

\section{Highlights}

- We present a simple method of stiff paste extrusion flow visualisation

- Static corner regions of unyielded paste are identified and sized

- Wall slip is seen on the die face and stick-slip flow noted on the extruder wall

- FEM simulations of the flow are carried out using a regularised fluid model

- The linear Navier wall slip model for VPF extrusion flow is assessed 


\title{
Investigation of static zones and wall slip through sequential ram extrusion of contrasting micro-crystalline cellulose-based pastes
}

\author{
M. P. Bryan, S. L. Rough, D. I. Wilson* \\ Department of Chemical Engineering and Biotechnology, University of Cambridge, Pembroke Street, Cambridge, CB2 3RA, UK
}

\begin{abstract}
The shape and size of static regions in the extrusion flow of a viscoplastic material through axisymmetric square entry dies was studied experimentally using a simple flow field analysis technique. A white micro-crystalline cellulose/water paste was extruded sequentially with a graphite-stained black analogue paste, revealing static regions in the corners of the system extending less than $30 \%$ of the barrel radius into the centre of the channel. Slow (relative to the bulk) slip flow along the die face was evident in the experiments, implying the existence of a stick-slip transition on this surface.

Finite element simulations using the Papanastasiou regularised Bingham plastic viscosity model and the linear Navier wall slip law were found to reproduce the experimental results poorly, capturing only separately the slip dominated bulk flow and the static corner region for different values of the slip coefficient $\beta$. The effect of severe wall slip was to reduce the distinction between unyielded and yielded material making the definition of the yield surface challenging. Investigation and implementation of a stick-slip wall condition is believed to be required for further work in this area.
\end{abstract}

Keywords: viscoplastic fluid, micro-crystalline cellulose, ram extrusion, wall slip, static zone, yield surface

\section{Introduction}

The extrusion of soft solids is a forming operation used for a variety of products such as catalyst monoliths [1], pasta [2], and pharmaceutical tablets [3]. The yield behaviour of soft solids allows the product of extrusion to retain its formed shape, and to be later hardened through processes such as drying or sintering.

Despite wide ranging applications, the precise deformation history of an extruded product is still difficult to calculate, due primarily to the complex flow patterns and rheologies of the materials used. Knowledge of this deformation history is critical to the optimisation of extrusion processing, particularly in the design of suitable equipment and the prevention of product defects.

Lab-scale analysis commonly utilises ram extrusion [4],

*Corresponding author, diw11@cam.ac.uk, +44(0)1223 334791 wherein the flow through the orifice (termed a die) is driven solely by a moving plunger (ram), as in a syringe. Ram extrusion contrasts screw or screen extrusion in which the flow is driven by mechanically actuated augers or rollers with a more complex flow pattern. Due to the confinement of the material by the equipment, direct visualisation of extrusion flow is difficult. As such, experimental measurement of an extrusion process is usually limited to the driving pressure force, the extrusion speed and the extrudate quality.

Stiff pastes are a class of viscoplastic fluids exhibiting large yield stresses. They can be processed through square or conical entry ram extrusion dies in which the contraction is abrupt $\left(90^{\circ}\right)$ or tapered, respectively. In the square entry case, static areas of unyielded material have been observed to form at the point of contraction, causing the flow field to naturally approximate a tapered system [5]. 
In contrast to extrusion with straight conical walls, the boundary of this static region is found to curve away from the extruder axis, towards the corners of the contraction (shown diagrammatically in part of figure 1). The location of this boundary cannot be predicted a priori, and hence the true form of the extrusion geometry is effectively unknown.

Stiff paste flow is further complicated by the occurrence of severe wall slip in most systems [6] in a departure from the no-slip condition common to most simpler, less viscous fluids. The no-slip condition is still obeyed on relatively short length scales, but the material is seen to effectively slip due to a thin layer (approximately 10 particle diameters thick for a solid-liquid suspension) of low viscosity material at the wall [7]. This liquid-rich phase acts as lubrication for the bulk flow, reducing the overall wall shear stress and creates the macroscopic appearance of slip.

It is difficult to directly observe the thin slip layer without affecting its thickness and composition, and as such, wall slip measurements are usually carried out macroscopically. A thorough review of wall slip in the context of rheometry was conducted by Barnes [7], who concluded that quantification of slip was crucial for accurate determination of shear stresses and subsequent material properties.

Unfortunately, the coupling of high yield strength and severe wall slip precludes normal rheometric analysis. As noted by Wilson and Rough [8], standard rheometric flows for stiff pastes exhibit measurement artefacts arising from slip and other effects. Moreover the force required to deform such materials is frequently outside the capacity of standard rheometers, assuming that slip itself does not dominate over deformation. If deformation is enforced using roughened surfaces to prevent slip, and the machine is sufficiently powerful, sample fracture often results rather than flow.

Other techniques must hence be employed to obtain information about stiff paste flow. Flow visualisation of ram extrusion can be used to gain some insight into the shear history of the extruded material and the form of the flow field. A simple example is the work of Green [9], who studied the deformation of plasticine (a viscoplastic material) as an analogue to metal forming processes. By marking the material sample with a regular grid and applying certain deformations to it, Green [9] was able to identify regions of compression, extension and shear in a variety of geometries, including extrusion through dies.

Wilmot et al. [10] utilised an hemicylindrical barrel and die system with quartz glass windows to track the flow of an extruding wax-based tungsten carbide paste. The results allowed a real time velocity profile to be calculated in the convergent zone of the extruder. Han and Ahn [11] limited the windowing to the flow after deformation, in the die land only, and imaged the flow of a concentrated alumina suspension using $10 \mu \mathrm{m}$ polymethyl methacrylate tracer spheres. In both cases, and for this style of flow visualisation in general, the validity of the results depends largely on whether the change in the system geometry significantly affects the flow field.

Recently, complex tomographic techniques to image extrusion flow fields have been used, namely magnetic resonance imaging (MRI) and positron emission particle tracking (PEPT). MRI techniques, as reported by Götz et al. [12, 13], Mantle et al. [14], Barnes et al. [15], image the flow through the movement of liquid in the extruding mass, or the paths of tracer particles added to the material during mixing. PEPT, as used by Wildman et al. [16] in their study of an extruding clay paste, tracks the decay of a radioactive isotope by gamma ray emission, which is typically introduced to the material through a suitably doped tracer particle.

Both MRI and PEPT allow in-situ non-disruptive measurement of the flow field, but are limited by the restrictions on materials that can be used. In the case of MRI, the extrusion equipment must be non-ferromagnetic due to the strong magnetic fields in the detector. Furthermore, 
the equipment must physically fit inside the magnet and still be operational. PEPT shares a similar limitation in that the system must be sufficiently transparent to gamma radiation. Wildman et al. [16] achieved this through use of non-metallic tools and low density extrusion material in order to minimise signal attenuation.

Simulations of stiff paste extrusion processes are not commonly reported in the literature, perhaps due to the lack of reliable experimental validation noted above. More frequently reported are simulations of benchmark flows for less stiff viscoplastic materials, although the inclusion of wall slip in such systems is less common. A rare study to include both non-Newtonian behaviour and wall slip in an extruder geometry is that of Mitsoulis and Hatzikiriakos [17] for a compressible PTFE paste. They used a Carreau fluid modified with additional structure parameters to model yield.

Horrobin and Nedderman [18] used elasto-plastic solids modelling to test the ideal, homogeneous deformation assumption inherent in the semi-empirical Benbow-Bridgwater extrusion model [19]. Their findings showed how the ideal deformation work must be modified to account for redundant internal shear, though their progress was hindered by the inability of their simulation code to model ratedependent plasticity common to stiff pastes.

Patel et al. [20] modelled a particulate paste as a modified Cam-Clay soil, with particular focus on liquid phase migration (LPM) (a filtering process in which the suspending liquid flows independently of the solids in the paste). Their conclusions, while largely focused on the LPM effects, suggest that this method of simulation was successful in capturing the unsteady behaviour of extruding stiff pastes, which were sensitive to both velocity and geometry. Despite this success, the authors are unaware of any further work using the soil mechanics approach to model extrusion.

Jay et al. [21] presented fluid mechanics based simulations for extrusion through axisymmetric square entry and conical geometries, using a biviscosity approximation for the yield behaviour of the material. Their conclusions focus on the strong effect of the characteristic Bingham number (see section 2.2) on the structure of the flow field and the pressure drop across the extruder. However, their simulations were conducted with a no-slip wall condition deviating significantly from stiff paste behaviour.

This paper presents observations from a simple method of flow field analysis, namely sequential ram extrusion of two physically identical but differently coloured pastes. This process is similar to that used by Ariawan et al. [22] as a coarse estimation of the flow field in PTFE resin extrusion, and by Perrot et al. [23] for cement-based materials. While this method leaves does not change the geometry of the flow field, it is limited to observing the state of the system at the end of a given extrusion and is not able to capture transient effects.

The experimental section is followed by an assessment of the difficulties in simulation of square entry ram extrusion, and is in effect a continuation of the work of Jay et al. [21] (albeit with the regularised fluid model) through the addition of wall slip to the simulation and its influence on static zone shape. The simulations presented here are not intended to replicate the experimental flow directly, owing to a lack of knowledge about the complex interactions involved in stiff paste extrusion. Instead the geometry is used as a starting point, and information about the real flow field used for general comparisons to the simulated cases.

\section{Methods}

\subsection{Experimental}

A stiff paste consisting of powdered micro-crystalline cellulose (MCC, Avicel PH101, FMC Corporation, Ireland) and deionised water was mixed to a solids weight fraction of $45 \%$ in a Kenwood planetary mixer (Kenwood Ltd., UK). The mixing protocol followed that of Zhang 
et al. [24], forming a white, easily deformable, granulated paste. The paste has been found by Zhang et al. [24] to behave reproducibly in square entry ram extrusion.

A contrasting black MCC paste was created separately using an identical mixing protocol, with the replacement of 2 wt.\% MCC by graphite powder (Fisher Scientific Ltd., UK). After mixing, both pastes were stored in sealed bags for a period of approximately two hours to allow equilibration of water within the granules. As will be shown in section 3.1, the extrusion behaviour of both pastes was found to be nearly identical.

Extrusions were performed using a Zwick-Roell Z050 computer controlled strain frame (Zwick GmbH \& Co., Ulm, Germany) modified to operate as a vertical ram extruder. A cylindrical $25 \mathrm{~mm}$ diameter barrel and a 3.5 $\mathrm{mm}$ diameter single-holed circular die of $24 \mathrm{~mm}$ length were used for the majority of the tests. Both the barrel and die were constructed from machined stainless steel. All tests used a $25 \mathrm{~mm}$ diameter, polyether ether ketone (PEEK) tipped steel rod as a ram, which was driven under displacement control by the strain frame.

The paste granules were loaded into the extrusion barrel by hand, to a total mass of $38 \mathrm{~g}$, compacted in $5 \mathrm{~g}$ increments using an iron bar to a height of approximately $68 \mathrm{~mm}$. The paste plug was further pre-compacted using the strain frame and a blanking plate to a peak force of $450 \mathrm{~N}$ (equivalent to $1 \mathrm{MPa}$ applied pressure), after which the blank was removed and replaced with the extrusion die.

For conciseness, it is convenient to define several terms, also seen in figure 1:

- Contrast paste - the material first loaded into the barrel, and hence the paste expected to extrude first

- Secondary paste - the material loaded into the barrel after the contrast paste

- Fill ratio - the percentage of the pre-extrusion plug by mass consisting of contrast paste

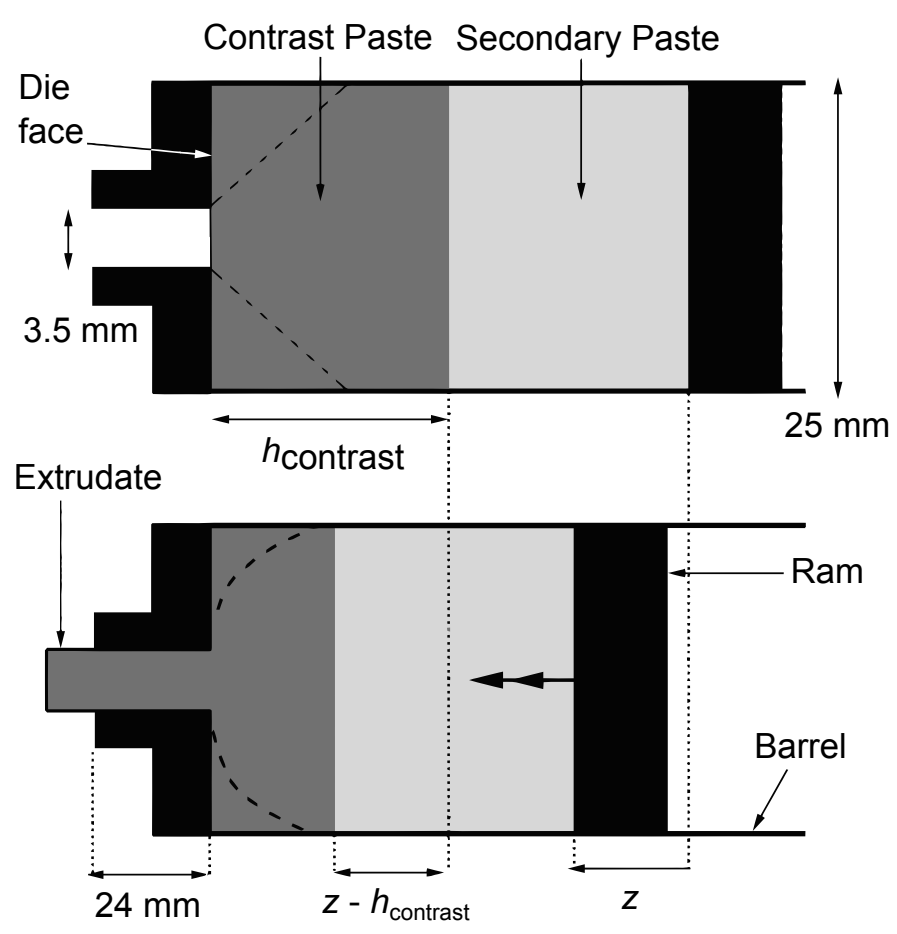

Figure 1: Diagram of the contrasting paste extrusion process. Upper diagram is prior to flow, lower diagram is during flow but before deformation of the interface. Dashed lines indicate potential static zone shapes, in the upper case as assumed by radial flow models such as Basterfield et al. [25].

- Die face - the surface of the extrusion die in contact with the paste, excluding the orifice

- Contrast paste height, $\left(h_{\text {contrast }}\right)$ - Initial length of contrast paste plug $($ fill ratio $\times 68 \mathrm{~mm}$ )

- Ram travel, $(z)$ - distance over which the ram moves towards the die face, controlled by the strain frame (range: $0-68 \mathrm{~mm}$ )

- Ram travel relative to initial contrast paste height, $\left(z-h_{\text {contrast }}\right)$ - Height of contrast paste remaining assuming no deformation of the interface

Fill ratios of $8,12.5,25,50 \%$ were used. Tests were performed with either black or white paste loaded initially in order to confirm that the results were independent of material type. Throughout, extrusions were performed at a constant ram velocity of $1 \mathrm{~mm} / \mathrm{s}$. The total ram travel in the course of an experiment varied from $20-60 \mathrm{~mm}$, 
with the resulting extrudates being collected for analysis. In order to verify that during filling a clear boundary between the two pastes was formed, several extrusions were performed in the absence of a die, such that an undeformed plug was ejected from the barrel.

After extrusion, the paste remaining in the barrel was carefully removed from the system and divided across its diameter using a scalpel. Each part of the plug was then photographed and measured for later analysis.

Measurements of features on the divided plug and confirmation of dimensions were performed using the software package ImageJ [26], using the diameter of the plug as a scale reference.

\subsection{FEM simulations}

Simulation of the flow was carried out using ANSYS Polyflow 14.5 (ANSYS, Inc., Pennsylvania, U.S.A.), a finite element based fluid dynamics software package designed for non-Newtonian fluid simulation. It was verified that inertial terms could be neglected due to the slow, high viscosity flow in extrusion and all simulations are solutions to the Stokes equations only.

The standard Galerkin formulation of the finite element method (FEM) requires that all functions in the PDEs to be solved are continuous in their first derivative. The inclusion of yield in viscoplastic fluid models violates this constraint, as the first derivative of the stress/shear rate rheometric curve is discontinuous at the yield point. Therefore other techniques must be used to circumvent this requirement, a review of some of which can be found in Dean et al. [27].

One such method is the Papanastasiou [28] regularised Bingham plastic viscosity model (equation 1) which uses a smoothing function to remove the discontinuity, and has the form:

$$
\eta(\dot{\gamma})=K_{\mathrm{BP}}+\tau_{\mathrm{Y}}\left(\frac{1-\exp (-c \dot{\gamma})}{\dot{\gamma}}\right)
$$

where $\eta$ is the apparent viscosity, $\dot{\gamma}$ the shear rate, $K_{\mathrm{BP}}$ the
Bingham viscosity coefficient, $\tau_{\mathrm{Y}}$ the material yield stress and $c$ the regularisation parameter.

The magnitude of the parameter $c$ controls how closely the regularised model matches the analytical model at low shear rates. The result is an effective viscosity which is very sensitive to low shear rates, and is asymptotic to infinity at $\dot{\gamma}=0$. In contrast to the analytical Bingham plastic, the 'unyielded' regularised fluid will always shear under stress however slowly, due to its finite (albeit large) viscosity near the asymptote.

A $c$ parameter of $10,000 \mathrm{~s}$ has been shown to be sufficient to describe yielding with acceptable accuracy for no-slip systems Mitsoulis [29]. Due to the presence of slip, $10,000 \mathrm{~s}$ was chosen as an initial value but $c$ was further varied in orders of magnitude up to $10^{10} \mathrm{~s}$ to ascertain its effect in this system.

Yield surfaces were identified by the now common stress state condition noted in Mitsoulis [29]:

$$
|\tau|=\sqrt{0.5\{\tau: \tau\}}=\tau_{\mathrm{Y}}
$$

where $\boldsymbol{\tau}$ is the symmetric extra stress tensor, and : denotes the tensor inner product. As applied to a regularised viscoplastic model, there is a certain degree of error in this criterion, as the extra stress tensor is derived from the velocity field of the solution. As noted previously, regularised fluids still undergo shear motion, even when unyielded. The magnitude of the difference between the analytical and regularised velocity profiles is therefore critical to the error in the determination of yield surfaces.

The linear Navier slip model [30] was used to describe the wall behaviour, viz.:

$$
\tau_{\mathrm{W}}=\beta u_{\mathrm{slip}}
$$

linking a wall slip velocity $u_{\text {slip }}$, wall shear stress $\tau_{\mathrm{W}}$ and a wall slip coefficient $\beta$. The model relates the velocity tangential to the wall to the shear stress at the wall, such that for a low value of $\beta$ the flow is slip dominated with a uniform velocity profile in the bulk material. The noslip condition can be recovered using the Navier slip law 
with an arbitrarily large value of $\beta$ (here $\beta=10^{15}$ ), such that $u_{\text {slip }}$ is approximately equal to zero irrespective of the shear stress. The units of $\beta, \mathrm{kg} / \mathrm{mm}^{2} \mathrm{~s}$, are omitted throughout for clarity.

Simulations were performed in 2D using an axisymmetric formulation and geometry identical to the experimental system. The domain was discretised using an unstructured quadrilateral mesh with approximately 9000 elements, with the density biased towards the walls of the system and the convergent zone. The mesh itself can be seen in the results section as part of figure 9 and a brief mesh study is described in the Appendix. An unstructured mesh was utilised to allow greater freedom to bias mesh density towards regions with large gradients in the solution fields while minimising computational time.

A uniform velocity inlet was used to replicate the action of the moving ram, while the slip boundary conditions were applied homogeneously to the system walls (the barrel, die face and die-land wall). A zero gauge pressure outlet condition was applied to the die exit, under the assumption of low residual stresses in the unyielded extrudate.

The interpolation used was quadratic in velocity and linear in pressure, conforming to the standard Taylor-Hood element. The convergence criteria used was the Polyflow default of $0.1 \%$ relative error between iterations, this being defined as the largest change in a solution variable evaluated at every node divided by the largest absolute value in the same field.

The flow is characterised by the Bingham number, defined as the ratio of the yield stress to the viscous stress:

$$
B n=\frac{\tau_{\mathrm{Y}}}{K_{\mathrm{BP}} \times \frac{u}{2 R}}
$$

for a yield stress $\tau_{\mathrm{Y}}$ and Bingham viscosity coefficient $K_{\mathrm{BP}}$, with a characteristic flow velocity $u$ in a circular channel of radius $R$. The characteristic velocity was chosen as the ram velocity $u_{\text {ram }}$, and the channel radius as $R_{\text {barrel }}$ since behaviour in the barrel is of interest. This definition differs from that used by Jay et al. [21], who used the Bingham number evaluated for the downstream, die land flow.

In the absence of reliable material property data for most stiff pastes, including the MCC under study in this paper, Bingham numbers of $0.1,1,10$ and 100 were instead arbitrarily chosen to ensure the full regime of viscoplastic behaviour was investigated (these correspond to $B n=0.0001372,0.001372,0.01372$ and 0.1372 as defined by Jay et al. [21]). The underlying assumption is that if MCC can be described by the Bingham model, the real behaviour would lie somewhere within this spectrum. A Newtonian fluid of viscosity $1 \mathrm{~Pa} \mathrm{~s}$ was also simulated for a baseline comparison (effectively $B n=0$ ).

\section{Experimental}

\subsection{Method validation}

Figure 2a shows extrusion pressure profiles obtained for plugs of $100 \%$ black and white MCC pastes. The two materials behave identically in the initial compression stage (0-2 $\mathrm{mm}$ travel) and exhibit identical pressure-travel gradients during the extrusion, which are typical of liquid phase migration as reported previously for these pastes by Mascia et al. [31]. The force required to extrude the graphite-containing MCC paste is approximately $5-10 \%$ smaller than that for the white material.

This reduced force could be attributed to the lubricating effect of graphite powder, which is commonly used in metal forging operations to reduce friction [32]. Graphite in this case could promote wall slip at the surface of the plug, or alternatively lubricate the shear flow of MCC particles in the convergent zone. The force reduction could also be attributed to increased water availability in the MCC-graphite matrix. As the graphite particles do not absorb water (in contrast to MCC particles), the additional free water would effectively change the paste composition, reducing the material yield stress and increasing the degree of wall slip [33]. These results are corroborated by 


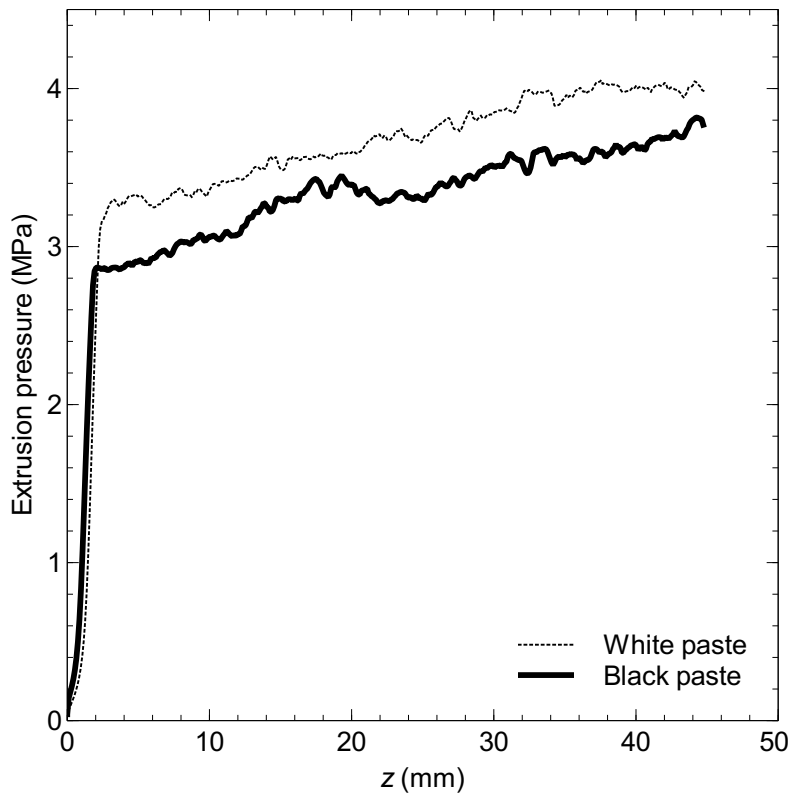

(a)

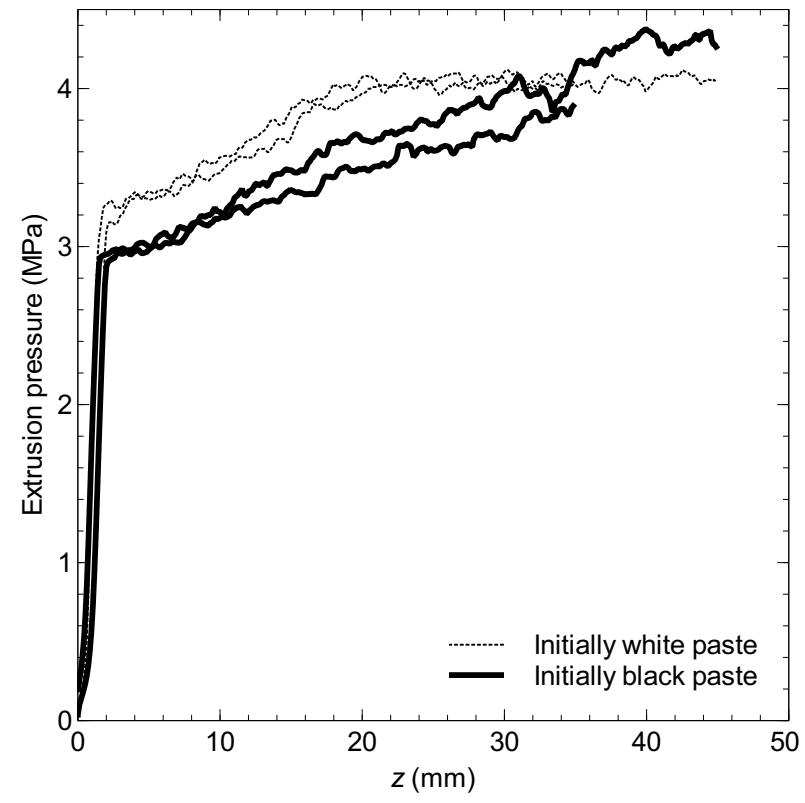

(b)

Figure 2: (a) Extrusion profiles obtained for $100 \%$ white and 100\% black MCC paste. (b) Extrusion profiles obtained for $50 \%$ fill ratio plugs with black-first and white-first loading.

experiments with $50 \%$ fill ratio plugs in both black-white and white-black fill orders. The extrusion pressure profiles are initially identical to figure $2 \mathrm{a}$, but merge to an average value after approximately $30 \mathrm{~mm}$ of ram travel (for a 68 $\mathrm{mm}$ plug), as seen in figure $2 \mathrm{~b}$. In the second part of each mixed fill extrusion, the extrudate consisted of a shrinking annulus of contrast paste surrounding a core of secondary material.

While the extrusion pressure differences indicate that the graphite does affect the paste material properties to a small degree, the primary concern of this work is the formation and persistence of static zones in the die entry region. Figure 3 shows the paste interface shape for both fill orders, demonstrating that there is no significant difference between the two configurations. The soft and heterogeneous nature of the material limits the reproducibility and accuracy of the technique so that such comparisons are partly subjective. If the presence of graphite provided additional lubrication in the corner regions, a noticeable change in the paste interface shape would be expected and

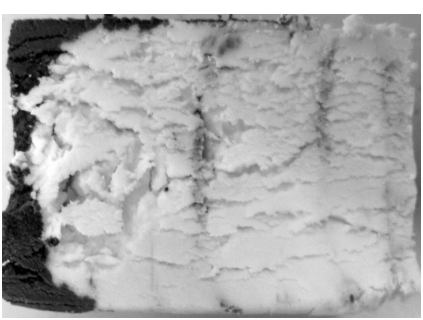

(a)

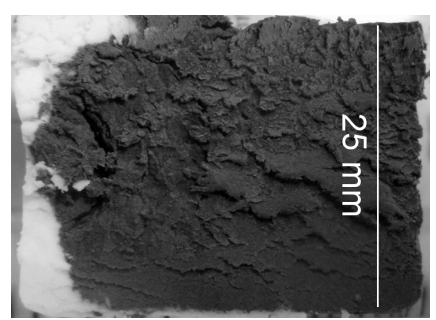

(b)
Figure 3: Comparison of contrast paste shape and area for $35 \mathrm{~mm}$ ram travel extrusions with (a) initially black, and (b) initially white paste.

this is not observed.

The extrusion pressure profiles for all experiments were (within natural variation) identical to those in figure $2 \mathrm{~b}$, and as such extrusion pressure will not be considered further in this paper.

\subsection{Boundary evolution}

It is possible to track the evolution of the boundary between the two pastes through interrupted extrusions over a range of ram travel values, and in doing so identify salient 
aspects of the velocity field. Areas of contrast paste which are replaced by secondary paste over time are by definition flowing, and the rate at which the front moves toward the die face gives an indication of the axial velocity component at a given point. It is, however, impossible to deconvolve the velocity field absolutely by this method as there is no mechanism by which paste moving radially can be tracked. Figure 4 shows the evolution of the boundary for $20 \leqslant z \leqslant 60 \mathrm{~mm}$. In the range $20-25 \mathrm{~mm}$, the boundary is seen to deform from nearly flat to curved, with the greatest deformation occurring towards the orifice along the axis of the plug. The remaining contrast paste is then observed to deplete, with areas adjacent to the orifice reducing in thickness faster than those in the corners. By $55 \mathrm{~mm}$ of ram travel, the boundary appears to be static, with contrast paste only occupying a small portion of the corner regions.

The position of the boundary was tracked along a line emanating from the corner at $45^{\circ}$ to the plug-end (see figure 5) which also shows, approximately, the final static zone shape. The results in figure 6 indicate that the contrast region reduces to a thickness of $2.5-3 \mathrm{~mm}$ for the largest $z-h_{\text {contrast }}$ values used. Front tracking along loci at other angles confirmed that the boundary was stationary at this stage (data not reported).

Expressed proportionally, the static zones in these instances were found to extend $20 \%$ of the barrel radius diagonally from the corner, and $30 \%$ of the same radius along the walls. It was also noted that for all $50 \%$ fill ratio experiments, a thin film of material (approximately $0.1 \mathrm{~mm}$ thick) persisted across the entire die face despite the depletion of the static region towards the corner.

The static zones observed here differ from images of the static zone reported by Wilmot et al. [10] for ram extrusion of wax-based tungsten carbide paste, which show the static area extending with a curved boundary to the orifice and beyond one barrel radius from the die face. Similarly, Perrot et al. [34] presented images of static zones formed from extruding kaolin paste with an approximately straight boundary extending many multiples of the radius along the barrel axis.

In contrast, Martin et al. [35] reported 'compacted' zones in a talc-based paste, which were of similar size and shape to the MCC static areas noted here, albeit with a straight boundary. The variation in the literature results suggest that the form of the static zone is determined primarily by the nature of the material being extruded.

\subsection{Wall slip observations}

The contrasting paste extrusion technique also allows some assessment of the wall slip behaviour of the material, seen as a moving boundary between contrast paste and secondary material at the barrel wall. Typically this boundary coincided with the internal boundary between the two pastes, however, on occasion thin smears of contrast paste were found to persist on the wall upstream of the internal boundary, as shown in figure 7. Thin layers of contrast paste were sometimes found a full barrel diameter upstream of the static region, long after the interface had fully deformed into the static region shape. These streaks were found to be variable and random across repeated tests, so that they could not easily be described quantitatively.

The heterogeneity observed could be interpreted as evidence for imperfect slip or stick-slip, which has not been considered to date in paste extrusion models such as those of Benbow and Bridgwater [19] and Basterfield et al. [25]. The very existence of the static zones noted previously also implies that some stick-slip boundary must exist, as the static material is by definition not undergoing slip flow.

Further evidence for this transition could be found on the end of the plug, i.e. that previously in contact with the die face, if sufficient secondary paste was made to flow through the orifice. The sequence of images in figure 8 shows the secondary paste breaking through a film of con- 


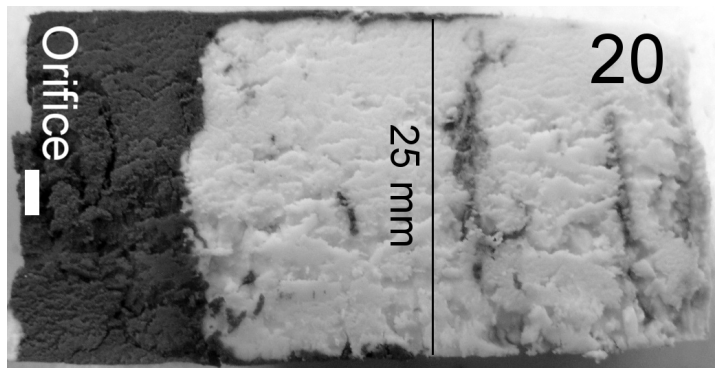

(a)

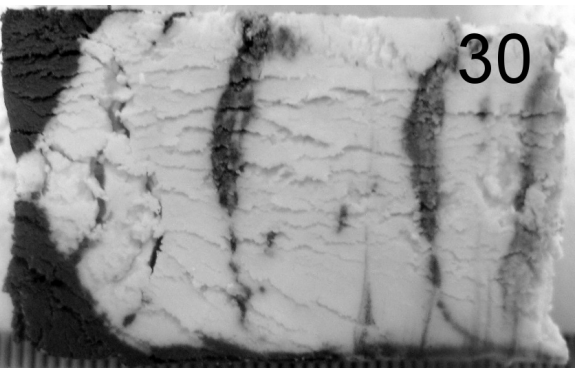

(c)

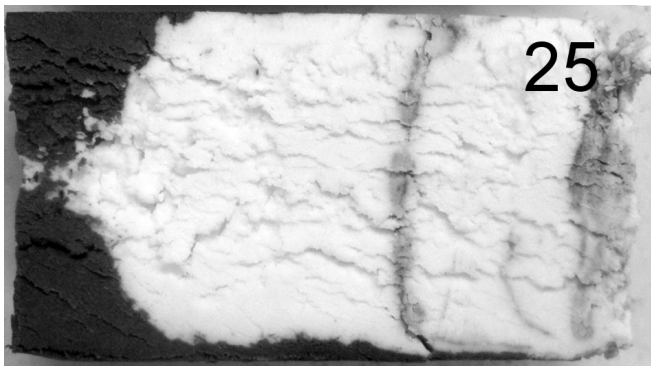

(b)

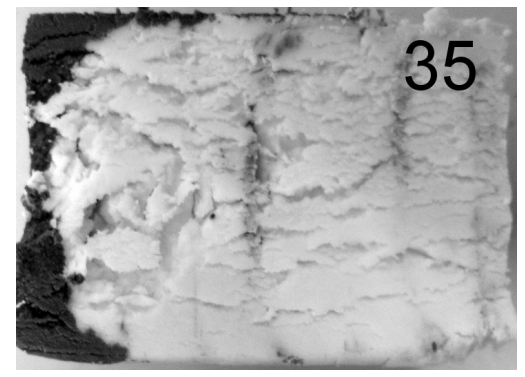

(d)

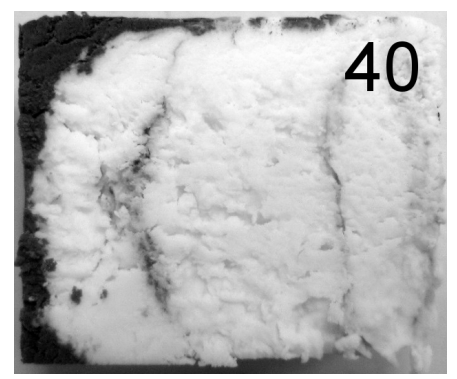

(e)

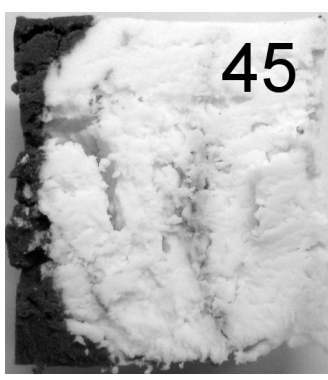

(f)

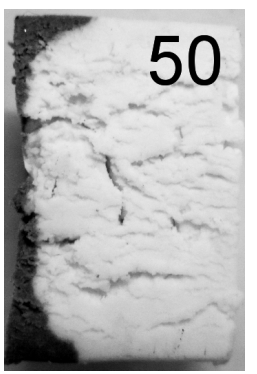

(g)

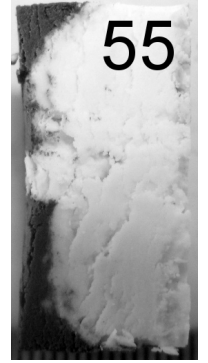

(h)

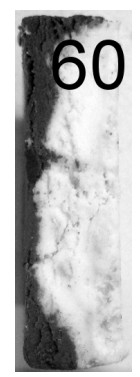

(i)

Figure 4: Progression of contrast paste boundary shape with increasing ram travel for $50 \%$ fill ratio. Figures (a) - (i) show 20 mm to 60 mm of ram travel in $5 \mathrm{~mm}$ increments displayed in the top right corner. Identical scale for all images. The white line in (a) also indicates the circular orifice diameter. 


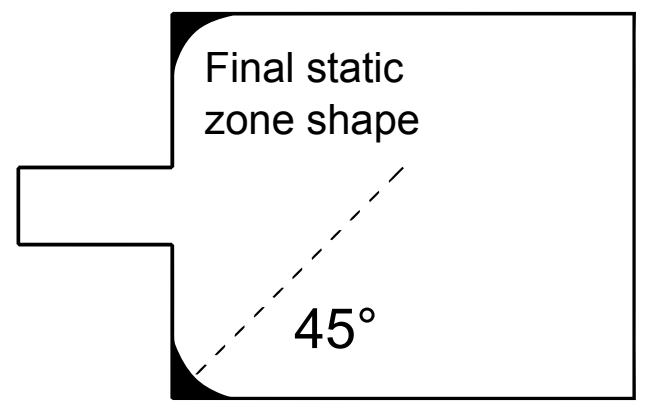

Figure 5: Schematic of the paste plug after extrusion showing part of the extrudate. The shaded region is the final static zone shape formed of contrast paste. The $45^{\circ}$ locus was used as a measure of the boundary thickness.

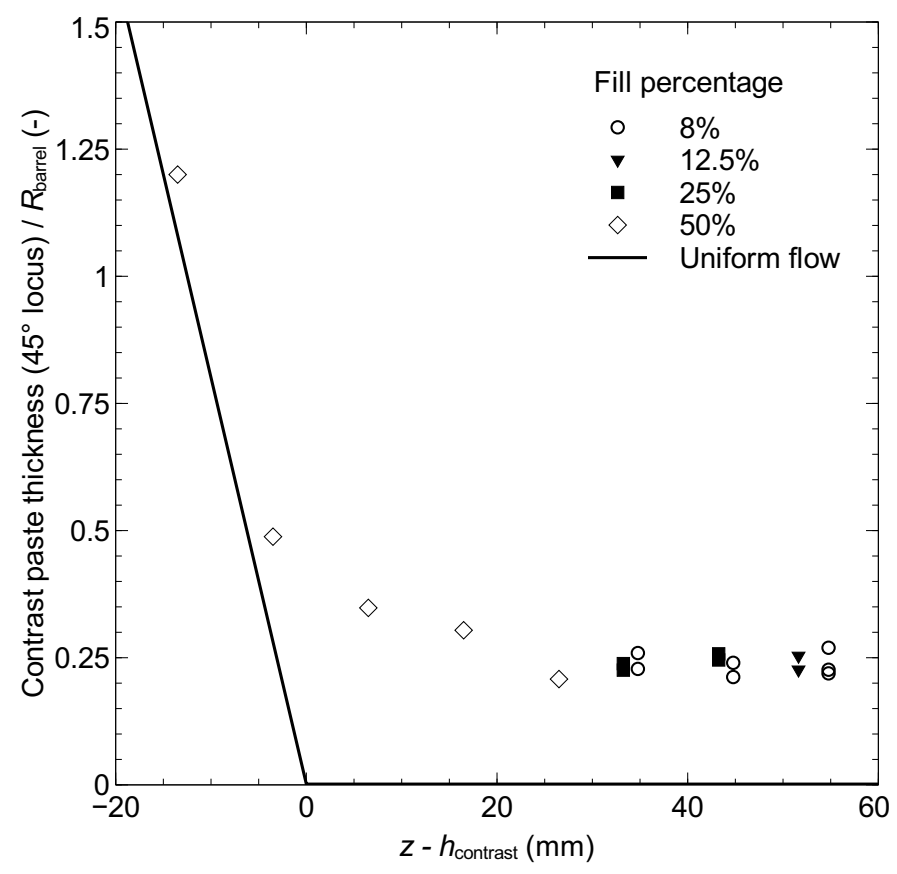

Figure 6: Size of contrast region along the $45^{\circ}$ diagonal locus, divided by the barrel radius $\left(R_{\text {barrel }}\right)$ for stainless steel tooling. The solid line labelled uniform flow indicates the diagonal extent if the boundary did not deform.

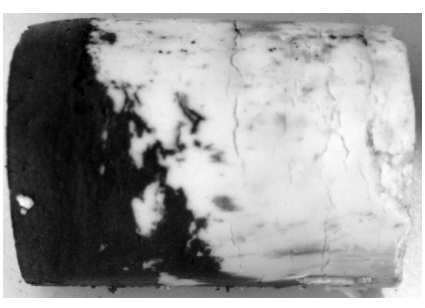

(a)

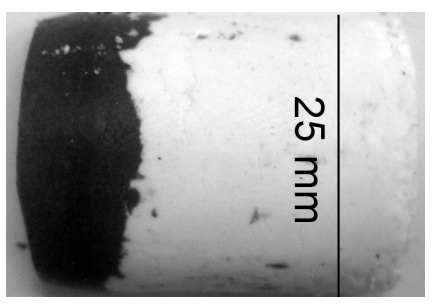

(b)
Figure 7: Comparison of contrast paste smear at the barrel wall for $z=35 \mathrm{~mm}, 50 \%$ fill ratio extrusion, showing inconsistency between separate extrusions.

trast material, revealing a region of slow slip flow (relative to the barrel wall) on the die face.

The slip area manifests as a mottled black/white pattern emanating radially from the orifice, confirming the existence of a radial flow field on the die face. The mottled pattern displays similar inconsistencies between tests as the slip on the barrel wall. The ring shaped structure of contrast material evident in figure $8 \mathrm{~d}$ marks the stick-slip boundary of the static region on this face.

It is possible that such behaviour is evidence of inhomogeneities in the paste plug composition during extrusion. If, as is commonly accepted in wall slip studies [36], the slip mechanism in the MCC system is dominated by the availability of free liquid at the wall, then regions of low liquid content could cause areas of low or even zero slip. In this case, the compaction of the plug in addition to the slow flow velocity in the corner region could cause a filtration condition, wherein the liquid filters from the corner into the extrudate, breaking the homogeneity of the material as in Rough et al. [37]. The resulting paste, being drier, may then exhibit no-slip behaviour in these areas.

\section{FEM simulations}

\subsection{Threshold slip models}

Having demonstrated the existence of stick-slip friction effects in stiff paste extrusion, it is apparent that simulation of the above material requires a wall slip model in 


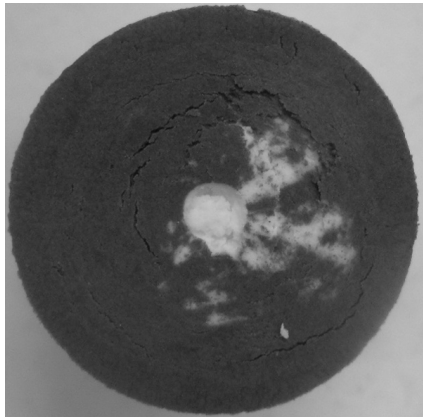

(a)

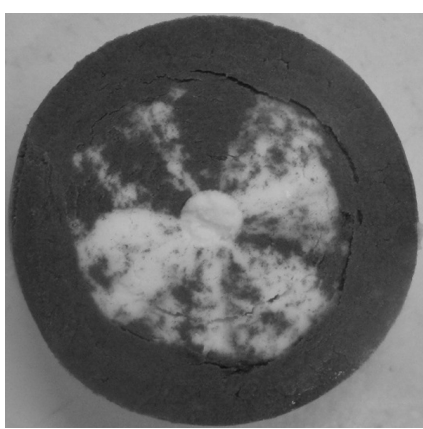

(c)

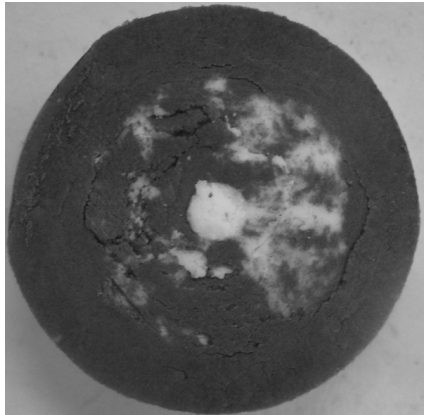

(b)

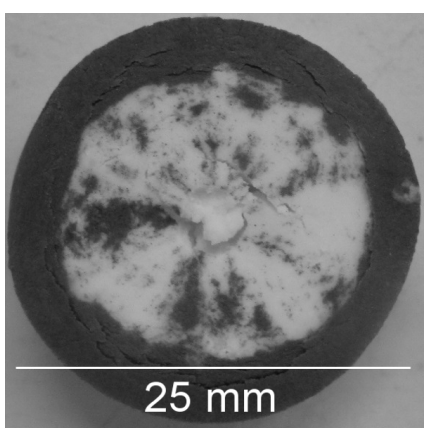

(d)
Figure 8: End-on view of plugs recovered from the barrel after experiment, showing secondary paste contacting the die-face. $z-h_{\text {contrast }}$ : (a) $33 \mathrm{~mm}$, (b) $43 \mathrm{~mm}$ (c) $51.5 \mathrm{~mm}$, (d) $54.6 \mathrm{~mm}$. which such a transition may occur. Such a slip law could take the following form, in which a threshold stress must be reached before slip initiates:

$$
u_{\mathrm{slip}}= \begin{cases}\left(-\tau_{\mathrm{W}, \mathrm{Y}}+\tau_{\mathrm{W}}\right) / \beta & \tau_{\mathrm{W}}>\tau_{\mathrm{W}, \mathrm{Y}} \\ 0 & \text { otherwise }\end{cases}
$$

for a wall slip-yield stress $\tau_{\mathrm{W}, \mathrm{Y}}$. The need to overcome a threshold stress is analogous to the Coulombic static friction coefficient in solid mechanics. The issue with use of equation 5 in standard FEM codes is its discontinuous gradient at the slip-yield point, which is an identical problem to that noted in section 2.2 for yield of viscoplastic fluids.

Roquet and Saramito [38] presented an FEM implementation that simultaneously solved analytically both the Bingham plastic model and a threshold slip function, based on the augmented Lagrangian-Eulerian method. They presented results for one dimensional flow, but the method has not yet been extended to two or three dimensional systems where the pressure field must be simultaneously calculated.

Other threshold-type slip models include those used by Rosenbaum and Hatzikiriakos [39] in the interpretation of slip and viscous-heating dominated capillary flow of molten polymers. The slip models used therein included a smoothed transition from slip to no-slip flow which is more suited to finite element modelling, a further example of which can be found in Damianou et al. [40].

Lacking the implementation of these models, an alternative is equation 3 , which describes what could be termed smooth linear slip, i.e. slip which increases monotonically from zero velocity at zero shear stress. This section investigates the deficiency of equation 3 in representing the behaviour seen experimentally, in order to demonstrate the need for further development in this area.

\subsection{Effect of slip on the static region}

Focusing primarily on the static corner region noted experimentally, yield surfaces are shown in figure 9 for four 
flows with different Bingham numbers over a range of wall slip coefficients $(\beta)$. A second yield surface not discussed further is that which bounds the central core of material, arising from partial yield of the bulk ( $c f$. pipe flow of a viscoplastic fluid). This is plotted for one case in figure $9 \mathrm{c}$.

In all cases, the yield surface furthest from the corner represents the no-slip state. For $B n=0.1$, the yield surface contours form loops which appear randomly distributed with $\beta$. These loops arise due to circulation zones in the velocity field at the corner, and imply that the flow is nearly entirely yielded. In contrast, the $B n=100$ case depicts a flow in which the fluid is (with respect to the regularisation function) entirely unyielded, except for a small convergent zone near the orifice. In reality such a material would be solid (and stationary) under these conditions: the regularisation function allows flow due to the finite (albeit large) viscosity it predicts. Such behaviour was noted by Moyers-Gonzalez and Frigaard [41] for duct flow of regularised viscoplastic fluids at the yield limit. Considering only the no-slip yield surfaces $\left(\beta=10^{15}\right)$ in the context of those reported by Jay et al. [21], there is good agreement for all of $B n=0.1,1,10$ shown here. However for the case of $B n=100$ in Jay et al. [21], which in our notation corresponds to $B n \approx 70,000$, the simulation does not generate the 'flow while unyielded' state seen in the present work, instead displaying a yield surface similar to but larger than $B n=10$ here. This discrepancy is likely due the different approximations of the yield condition in the bi-viscosity and regularised fluid models, rather than any fundamental difference in the flow.

The principal finding in figure 9 is that the slip function alters the shape and size of the static zone. For both $B n=1$ and 10, the boundary recedes towards the corner with increasing slip, and in some its curvature inverts from concave to convex. The unintuitive convex form was seen computationally in static zones reported by Taylor and Wilson [42] for duct flow of a viscoplastic fluid, which was further explored by Wang [43]. Wang [43] concluded that the convexity was a result of too small a value of the regularisation parameter $c$ and insufficient mesh resolution causing 'blurring of the yield surface'.

Figure 10 shows several solution variables at $B n=10$ measured along a $45^{\circ}$ line emanating from the static corner (see figure 5). It is immediately apparent that increasing slip effectively smooths the solution between the corner (expected to be static) and convergent zone (expected to be yielded and flowing). Rather than increased slip reducing the influence of the corner on the flow (as might be assumed from figure 9), the plots instead show that zone of influence is of similar size but faster flowing and less viscous. To test whether the smoothing exhibited by the slip dominated solutions was evidence of an insufficiently large $c$ parameter, the series of simulations was repeated using $c$ values in order of magnitude steps between the $10,000 \mathrm{~s}$ used above and $10^{10} \mathrm{~s}$. The results, not shown, indicated only a minimal effect, with most solutions being indistinguishable from each other for any given value of the slip coefficient.

Vector plots of the velocity field (also not shown) for the high slip cases show fluid which effectively follows the wall contour, changing direction rapidly at the corner. This rapid change manifests as a high shear rate, causing the viscosity near the corner to be nearly equal to that of the rest of the convergent zone. Despite the experimental system being slip dominated, the 'solid' unyielded zone seen therein is completely uncaptured by the combination of models in the simulation.

\section{3. 'Error' in the regularised velocity field}

As a measure of the 'error' in the velocity field due to the regularisation of the yield behaviour, the velocities at the yield surfaces for $B n=10$ in figure $9 \mathrm{c}$ were calculated and the values along the 45 degree locus extracted. It bears repeating that in reality these boundaries should represent the border between static, unyielded fluid and just-moving material. The data in figure 11 show this velocity as a 


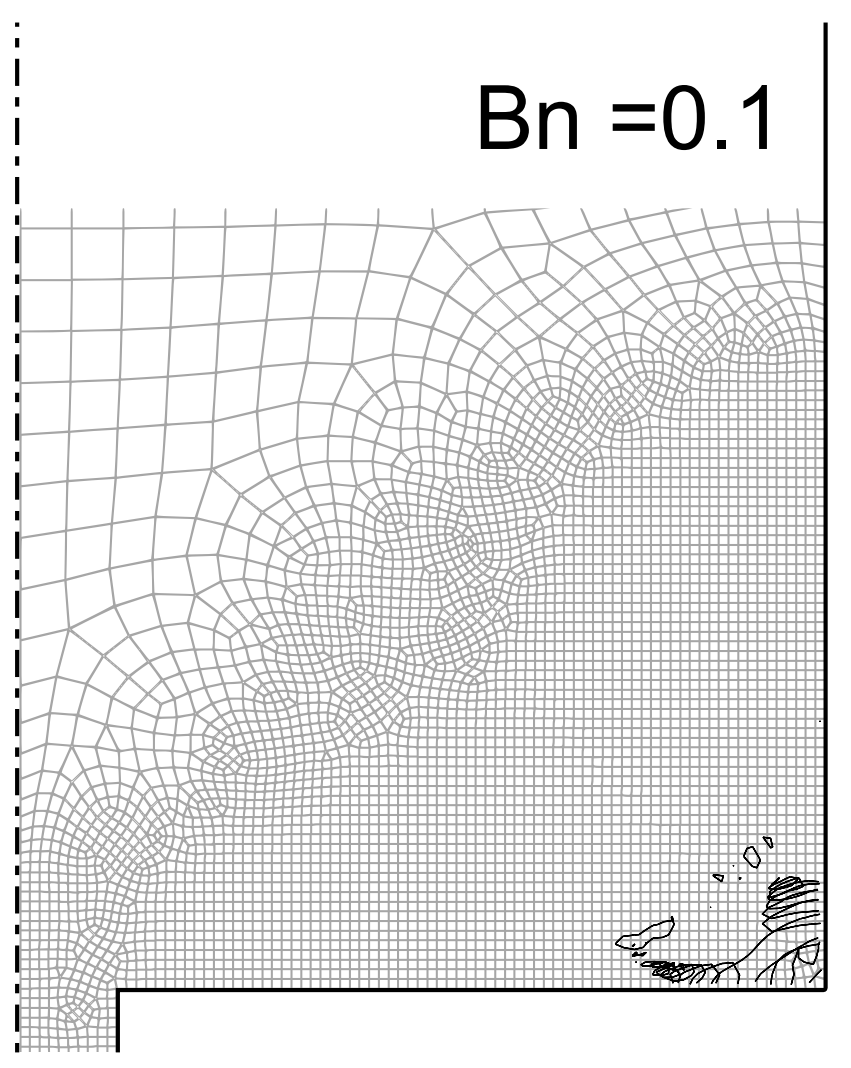

(a)

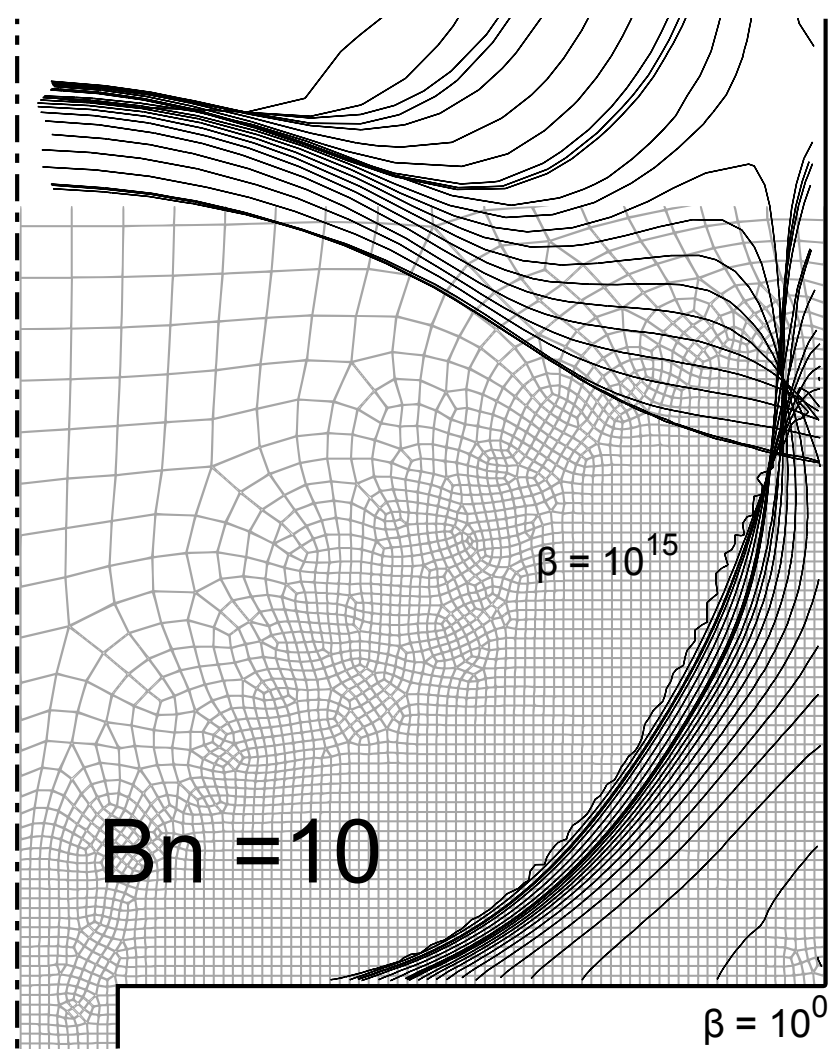

(c)

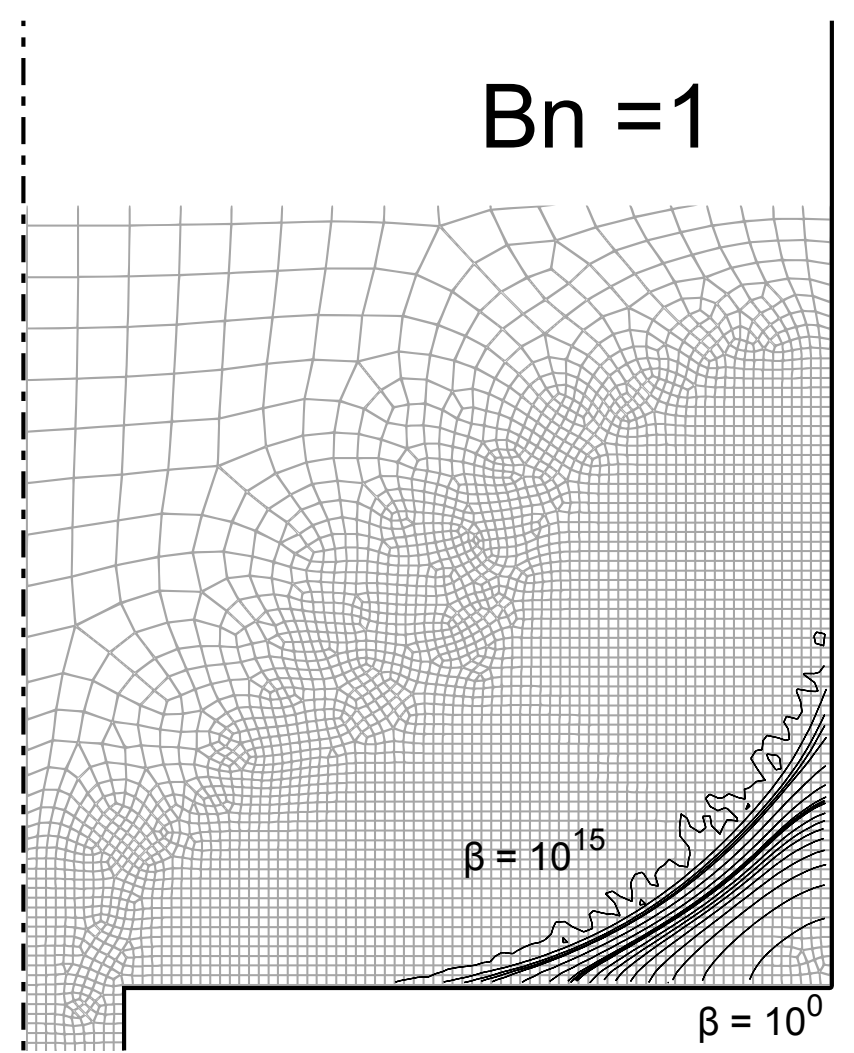

(b)

\section{$B n=100$}

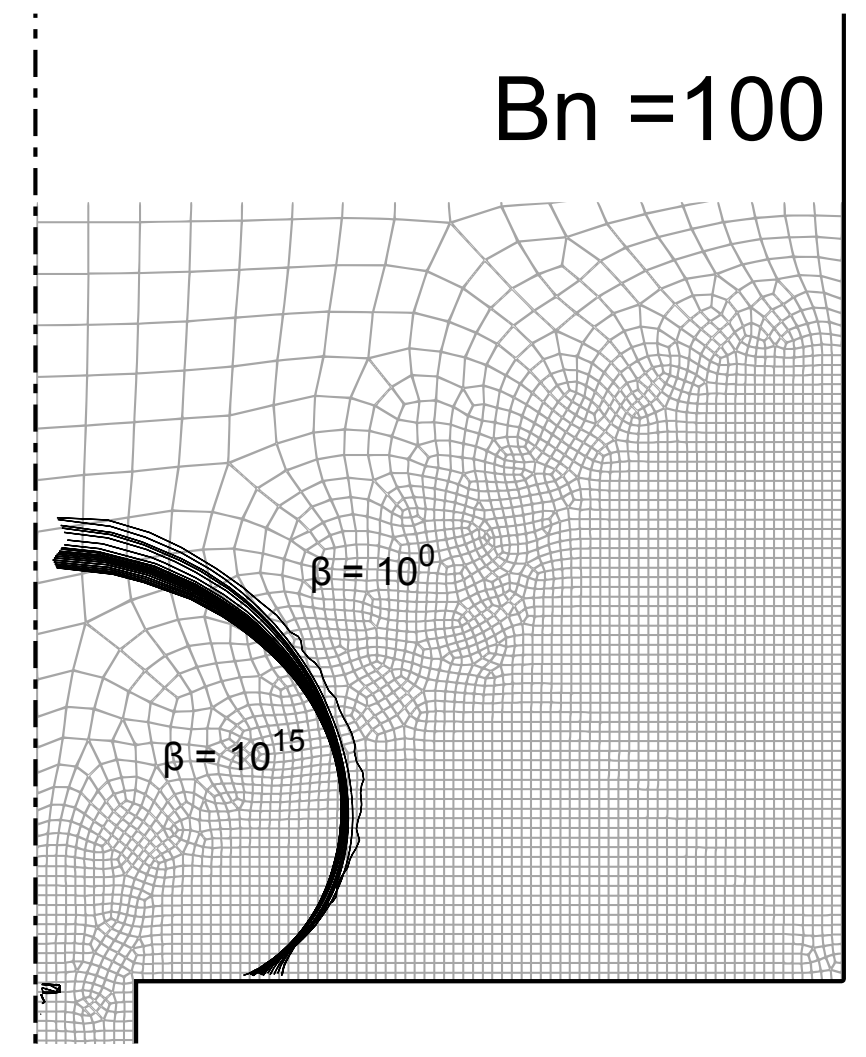

(d)

Figure 9: Static zone boundaries for (a) $B n=0.1$, (b) $B n=1$, (c) $B n=10$, (d) $B n=100$, in the range $\beta=1$ to $\beta=1 \times 10^{15}$. Boundaries further from the outer corner are those of larger $\beta$ values. Additional contours in (c) are the yield surfaces of the central plug. The bold curve in the corner of each figure indicates the experimentally observed statỉ zone boundary. Local mesh shown for reference. 


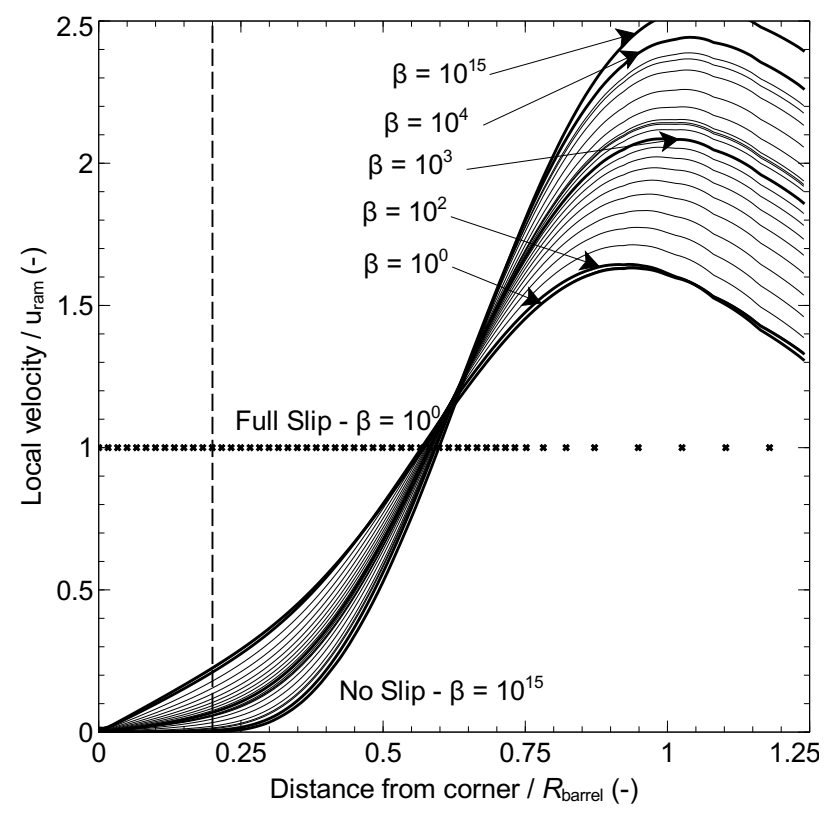

(a)

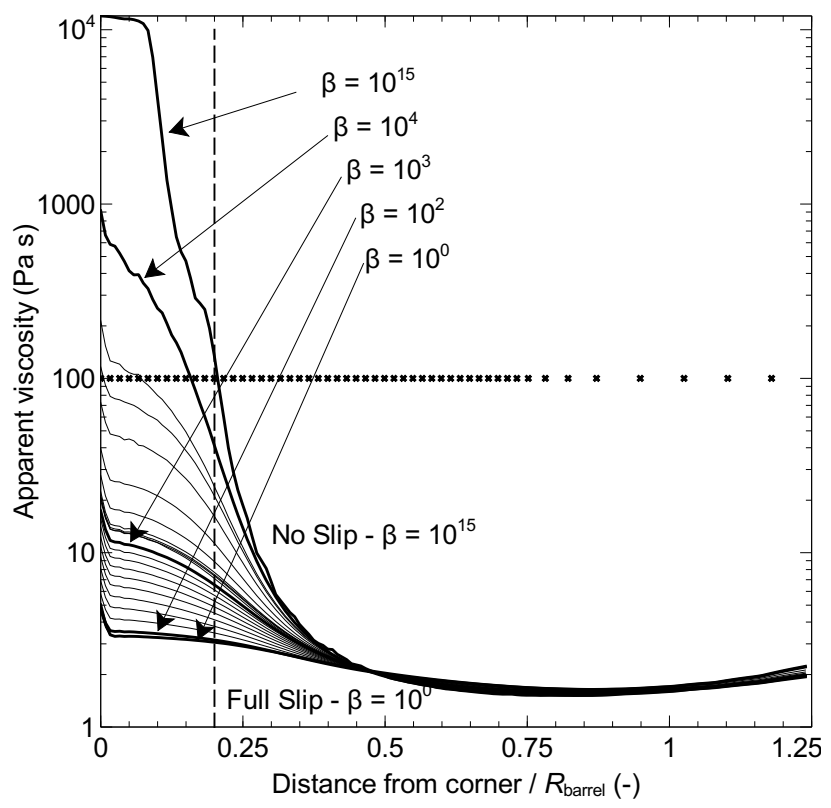

(c)

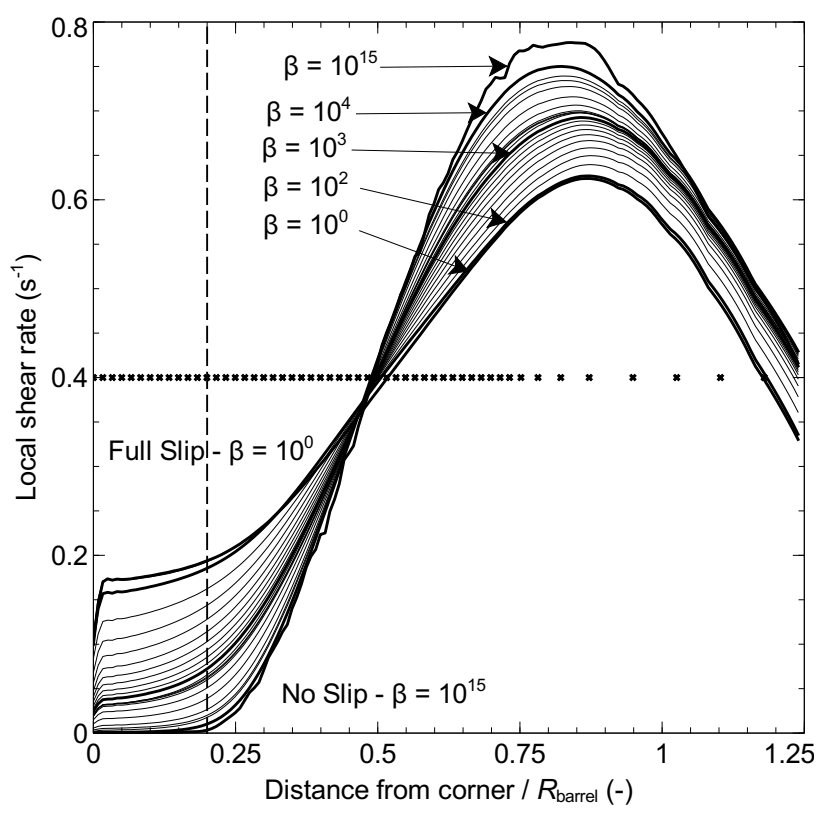

(b)

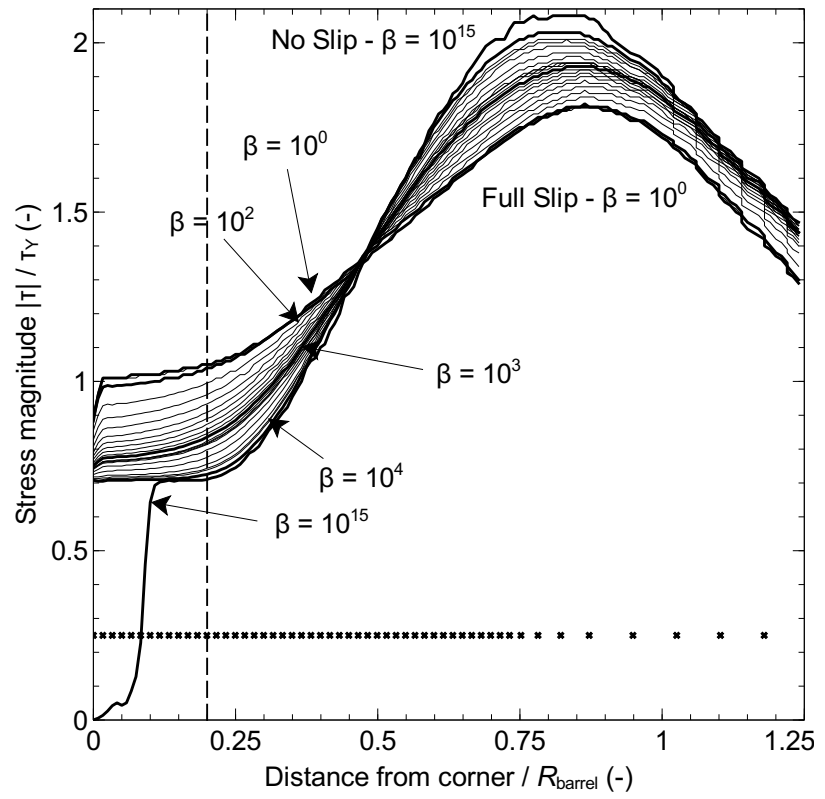

(d)

Figure 10: Plots of solution variables along the $45^{\circ}$ locus for $B n=10$ for (a) velocity / $u_{\text {ram }}$, (b) shear rate, (c) viscosity (logarithmic y-axis) and (d) stress magnitude / yield stress. Vertical dashed lines indicate the position of the experimental static zone boundary. Horizontal crosses indicate the local mesh spacing. 


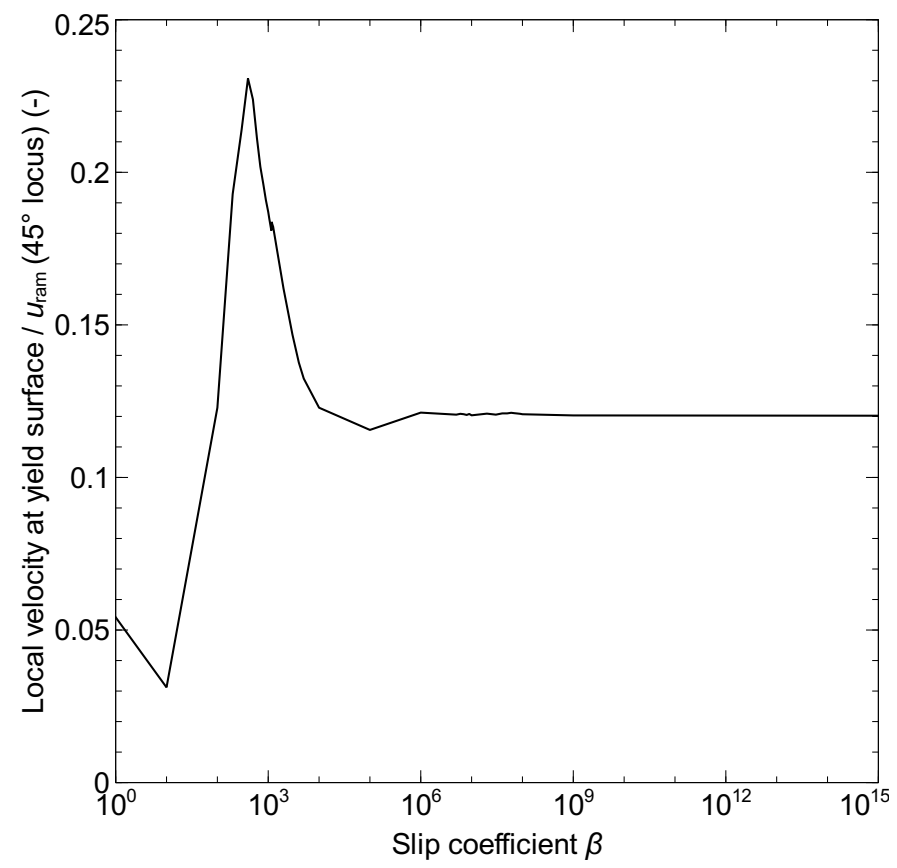

Figure 11: Velocity / $u_{\text {ram }}$ at the yield surface on the $45^{\circ}$ locus for $B n=10$ as a function of slip coefficient $\beta$.

function of slip coefficient $\beta$.

It is clear that even for the no-slip case, the static region boundary demarks an area which is flowing appreciably relative to the bulk. In the worst case, a high slip, low $\beta$ simulation, the fluid is moving at over $20 \%$ of the characteristic bulk velocity with an internal shear gradient yet is treated as completely unyielded by the stress magnitude criterion. This highlights the difficulty, almost a level of subjectivity, in defining a yield surface for regularised viscoplastic fluids in the presence of slip. Such difficulties, particularly for flows in which large portions of the material are unyielded (as in slip dominated flow) were noted and covered with substantial mathematical rigour by Frigaard and Nouar [44].

\subsection{Simulation outcomes}

The simulation results presented highlight that smooth slip functions do not combine favourably with a regularised fluid model for this application. The threshold, stick-slip type functions referenced above are perhaps a necessary modification that would generate the yield surfaces seen experimentally.

As an aside, it is worth considering more carefully the physical nature of slip in stiff viscoplastic systems such as the MCC paste studied here. For these materials, the duct flow behaviour is of an apparently unyielded body moving relative to the wall. All processes facilitating the flow occur in a thin layer immediately adjacent to the wall, such that it is impossible to discern whether the governing behaviour is of very localised yield or wall slip.

Indeed, it is arguable that the definition of apparent slip, a reduction in particle volume fraction resulting in a low viscosity layer, is not dissimilar to yield on a microscopic scale. In this sense, yield and slip are two expressions of the same physical behaviour. The issue in simulation therefore is the applicability of the continuum hypothesis on the small length scales concerned, and whether macroscopic yield models such as the Bingham plastic are appropriate for predicting slip-like, wall localised yield in stiff systems.

\section{Conclusions}

The contrasting paste extrusion technique, in which two differently coloured materials are sequentially ram extruded through a single axisymmetric circular orifice, was successfully applied to a micro-crystalline cellulose-based stiff paste. The method revealed the form of the static region at the corner of the ram extruder, which was found to extend only a small distance (20-30\% of the barrel radius) into the flow with a curved boundary. This small region is similar in form but smaller in size than the static areas imaged in other flow visualisation studies such as Barnes et al. [15] and Wilmot et al. [10].

This technique also revealed an area of slow, radial wall slip flow on the die face bounded circumferentially by the static region. The slip on this face, and between the barrel wall and the paste plug was found to be heterogeneous, i.e. parts of the interface underwent slip, while others stuck to the surface. This observation, and the fact that the static 
regions are by definition stuck to the extruder corner, allowed the conclusion that the paste undergoes stick-slip behaviour. Further experimental work would require the application of the method to other stiff pastes, in order to determine whether the features noted are specific to MCC.

It would also be possible to utilise an extrusion system with deliberately roughened walls, potentially eliminating slip flow, allowing the material's bulk rheology to solely determine the static zone shape and size. This was not carried out in the current study due to a lack of suitably roughened equipment.

FEM simulations were performed to demonstrate the deficiency of the linear Navier slip model in representing flows similar to that seen experimentally. The performance of the regularised Bingham plastic model and the linear Navier slip model was found to be poor, with the wall slip law interacting with the smooth viscosity function to reduce the distinction between yielded and unyielded fluid. In slip dominated systems, the velocity on the edge of the notionally 'static' regions was found to be approximately $20 \%$ of the bulk velocity, highlighting that yield surface definition is not absolute.

Future simulation work will focus on the rigorous description of the Bingham plastic (or other yield stress fluids), in addition to implementation of a threshold stick-slip model. Initial efforts will use an augmented LagrangianEulerian technique as reported by Saramito and Roquet [45], Huilgol and You [46], Muravleva et al. [47], which allows solution of the analytical material model.

\section{Acknowledgements}

The authors are grateful to Merck \& Co., Inc. for the supply of the powdered micro-crystalline cellulose used in these experiments, and to Sandvik AB and Ceratizit $\mathrm{GmbH}$ for jointly funding this project.

\section{Nomenclature}

\section{Roman}

Bn Bingham number

$c$

Papanastasiou regularisation parameter (s)

$h_{\text {contrast }}$

Initial length of contrast paste plug . (mm)

$K_{\mathrm{BP}}$

Bingham viscosity coefficient

$R$

Characteristic radius

( $(\mathrm{mm})$

$R_{\text {barrel }}$

Barrel radius

$(\mathrm{mm})$

$u$

Characteristic velocity $(\mathrm{mm} / \mathrm{s})$

$u_{\text {ram }}$

Ram velocity $(\mathrm{mm} / \mathrm{s})$

$u_{\text {slip }}$

$z$

Wall slip velocity $(\mathrm{mm} / \mathrm{s})$

Ram travel coordinate, positive towards die face . $(\mathrm{mm})$

$z-h_{\text {contrast }}$

Ram travel relative to initial contrast paste height $(\mathrm{mm})$

\section{Greek}

Wall slip coefficient $\left(\mathrm{kg} / \mathrm{mm}^{2} \mathrm{~s}\right)$

Local shear rate $\left(\mathrm{s}^{-1}\right)$

Apparent viscosity

$\|\varphi\|_{1, i}$ $\mathrm{L}_{1}$-norm of the solution field $\varphi$ for number of cells $i \ldots \ldots \ldots \ldots \ldots \ldots \ldots \ldots$ (as $\varphi)$

Symmetric extra stress tensor

$\tau_{\mathrm{W}}$

Wall shear stress

$\tau_{\mathrm{W}, \mathrm{Y}}$

Slip-yield stress

$\tau_{\mathrm{Y}}$

Bingham plastic yield stress 


\section{Appendix: Mesh convergence study}

In order to verify the suitability of the finite element grid, a series of simulations were carried out using four combinations of Bingham number $B n$ and slip coefficient $\beta$ representing the range of high and low slip and yield behaviour. The grid convergence criteria used were the $\mathrm{L}_{1}$-norms of the primal velocity and pressure fields, consistently evaluated at the nodes of the 2,438 element mesh. In order to display both criteria on the same plot, both were normalised by the respective value at the highest grid density $(121,200$ elements). The results of the mesh convergence study are summarised in figure A.1.

In all cases, the solution norm is constant above approximately 2,500 elements. For both no-slip cases, the pressure field norm attains its final value nearly immediately. A more dense mesh of 8,326 elements was used for all simulation results in this article due to the increased resolution permitted for determining the yield surface contours. As noted in the methods section, the mesh density was biased towards the walls of the simulation geometry and towards the convergent and corner regions.

\section{References}

[1] P. Forzatti, D. Ballardini, L. Sighicelli, Preparation and characterization of extruded monolithic ceramic catalysts, Catalysis Today 41 (1-3) (1998) 87-94, ISSN 09205861, doi: 10.1016/S0920-5861(98)00040-6.

[2] M. Lucisano, M. A. Pagani, M. Mariotti, D. P. Locatelli, Influence of die material on pasta characteristics, Food Research International 41 (6) (2008) 646-652, ISSN 09639969, doi: 10.1016/j.foodres.2008.03.016.

[3] P. J. Harrison, J. M. Newton, R. C. Rowe, Flow defects in wet powder mass extrusion, Journal of Pharmacy and Pharmacology 37 (2) (1985) 81-83, ISSN 2042-7158, doi:10.1111/j.20427158.1985.tb05011.x.

[4] D. I. Wilson, S. L. Rough, Exploiting the curious characteristics of dense solid-liquid pastes, Chemical Engineering Science 61 (13) (2006) 4147-4154, ISSN 00092509, doi: 10.1016/j.ces.2005.10.032.

[5] J. J. Benbow, E. W. Oxley, J. Bridgwater, The extrusion mechanics of pastes: the influence of paste formulation on extrusion parameters, Chemical Engineering Science 42 (1967).

[6] G. M. Corfield, M. J. Adams, B. J. Briscoe, P. J. Fryer, C. J. Lawrence, A critical examination of capillary rheometry for foods (exhibiting wall slip), Food and Bioproducts Processing 77 (1) (1999) 3-10, ISSN 09603085, doi: $10.1205 / 096030899532196$.

[7] H. A. Barnes, A review of the slip (wall depletion) of polymer solutions, emulsions and particle suspensions in viscometers: its cause, character, and cure, Journal of Non-Newtonian Fluid Mechanics 56 (3) (1995) 221-251, ISSN 03770257, doi: 10.1016/0377-0257(94)01282-M.

[8] D. I. Wilson, S. L. Rough, Paste engineering: Multi-Phase materials and multi-phase flows, The Canadian Journal of Chemical Engineering 90 (2) (2012) 277-289, ISSN 1939-019X, doi: $10.1002 /$ cjce.20656.

[9] A. P. Green, The use of plasticine models to simulate the plastic flow of metals., Philosophical Magazine Series 742 (327) (1951) $365-373$.

[10] R. B. Wilmot, R. Barbist, H. Ferstl, C. Gruber, W. Brandstätter, M. J. Patel, D. I. Wilson, Characterisation and design of wax-based hard metal extrusion processes, 17th Plansee Seminar, Plansee Group, Reutte, Austria 2 (HM15)

[11] W. Han, K. H. Ahn, In situ flow visualization of capillary flow of concentrated alumina suspensions, Rheologica Acta 52 (6) (2013) 547-556, ISSN 0035-4511, doi:10.1007/s00397-013-0710$\mathrm{x}$.

[12] J. Götz, H. Buggisch, M. Peciar, NMR imaging of pastes in a 


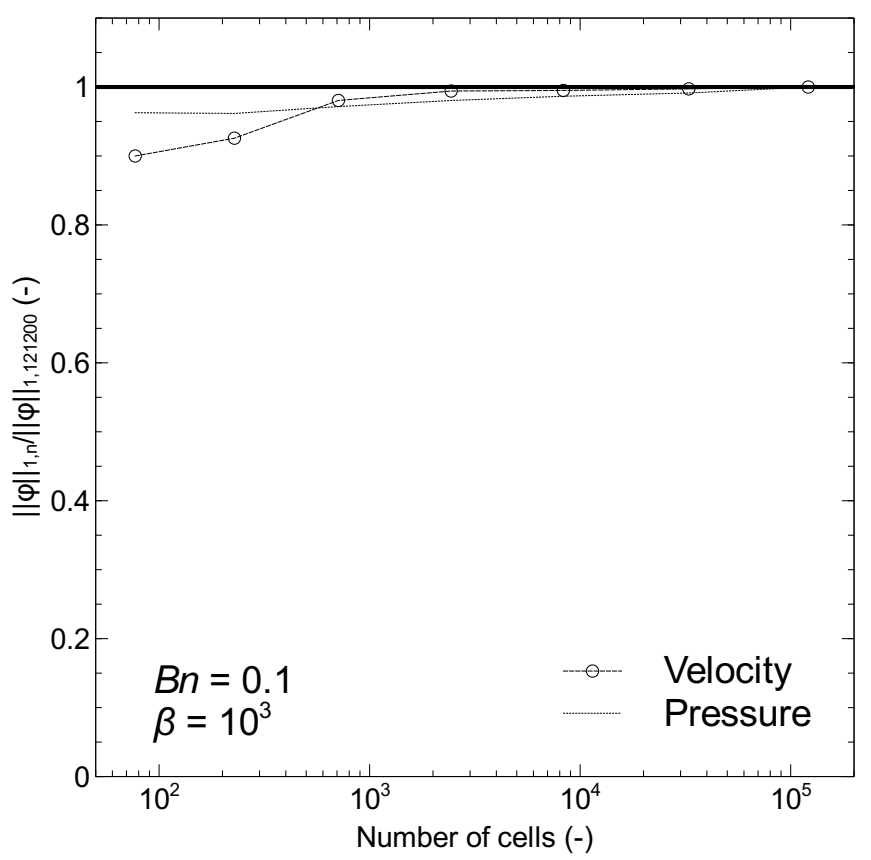

(a)

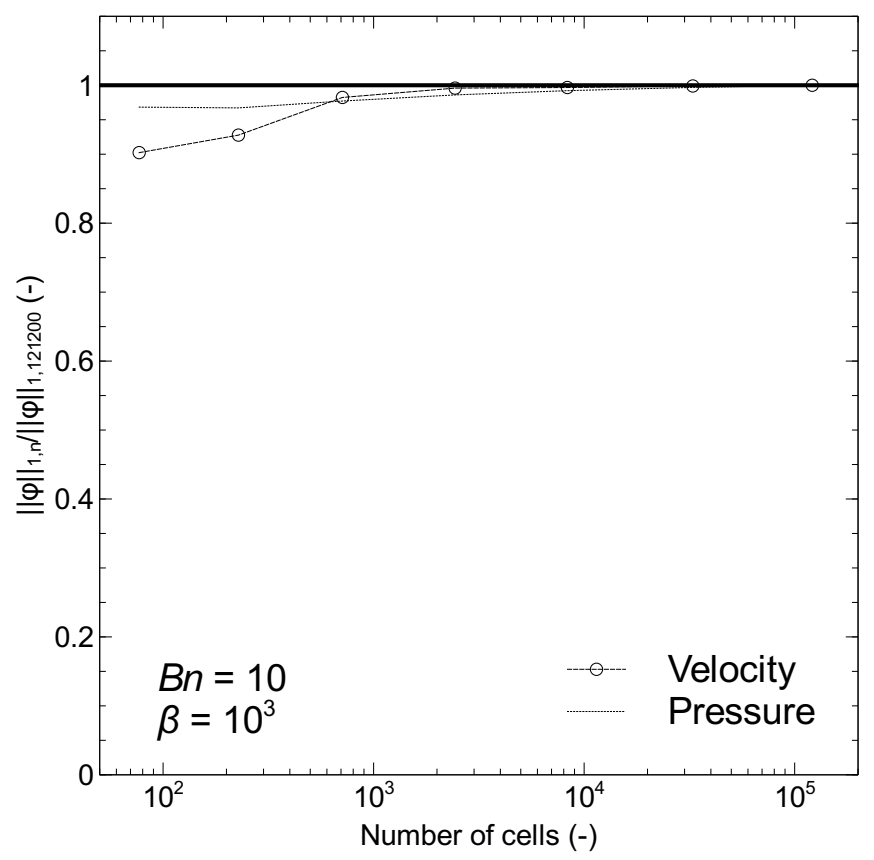

(c)

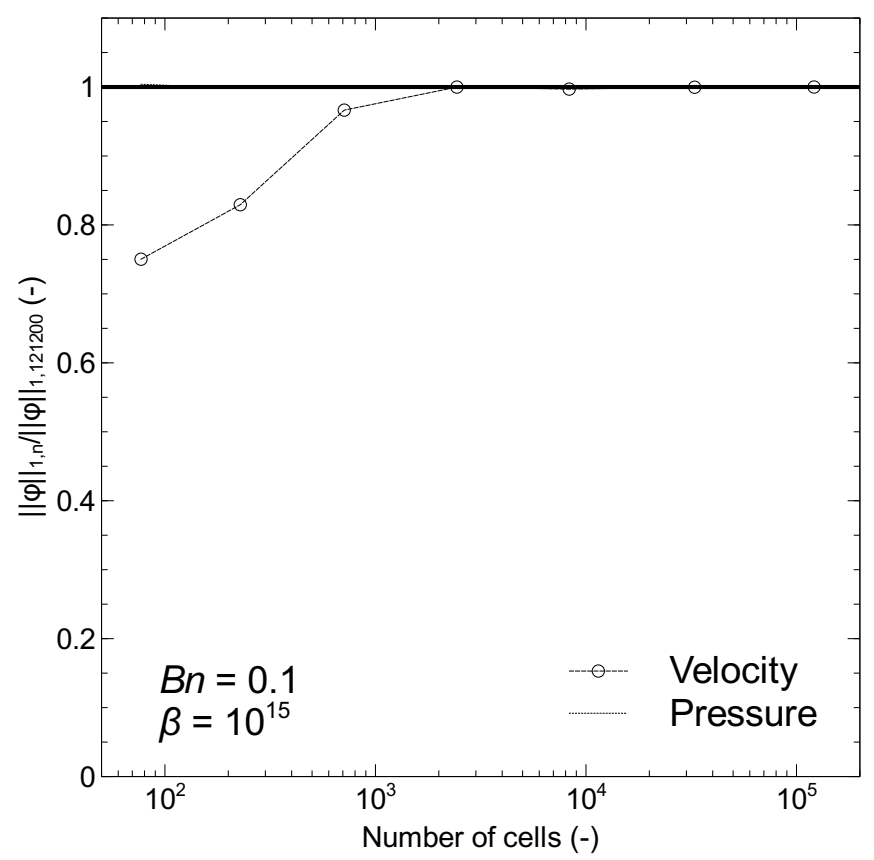

(b)

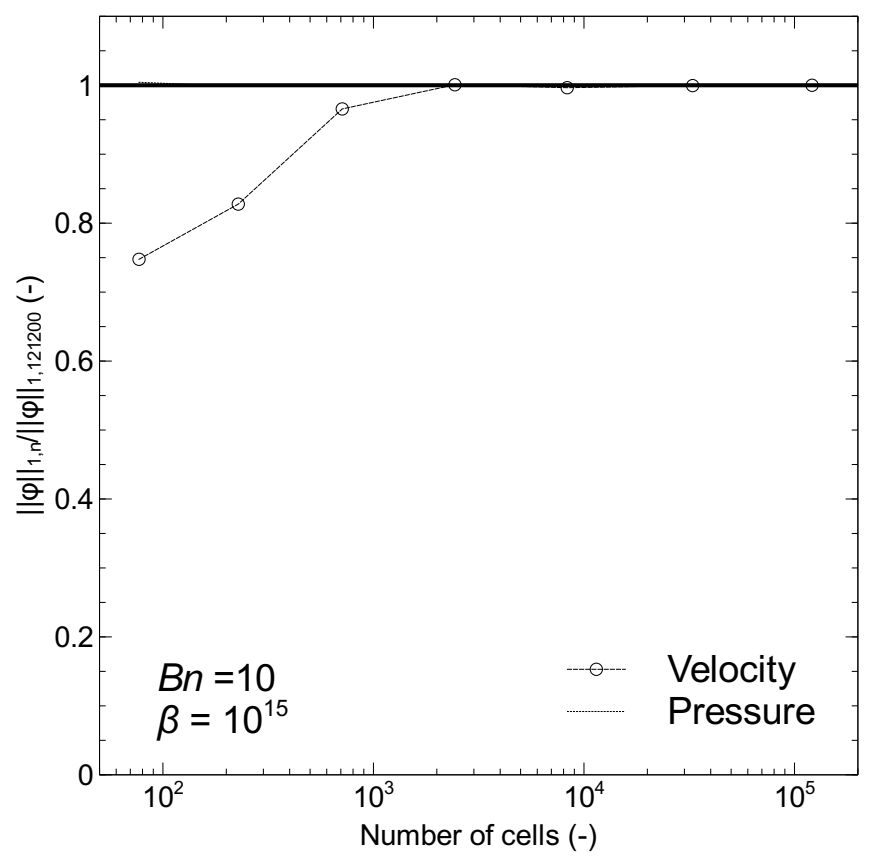

(d)

Figure A.1: Grid convergence for (a) $B n=0.1 / \beta=10^{3}$ (low yield strength, high slip), (a) $B n=0.1 / \beta=10^{15}$ (low yield strength, no-slip), (a) $B n=10 / \beta=10^{3}$ (high yield strength, high slip), (a) $B n=10 / \beta=10^{15}$ (high yield strength, no-slip). 
ram extruder, Journal of Non-Newtonian Fluid Mechanics 49 (1993) 251-275

[13] J. Götz, W. Kreibich, M. Peciar, Extrusion of pastes with a piston extruder for the determination of the local solid and fluid concentration, the local porosity and saturation and displacement profiles by means of NMR imaging, Rheologica Acta 41 (12) (2002) 134-143, ISSN 0035-4511, doi:10.1007/s003970200012.

[14] M. D. Mantle, M. H. Bardsley, L. F. Gladden, J. Bridgwater, Laminations in ceramic forming - mechanisms revealed by MRI, Acta Materialia 52 (4) (2004) 899-909, ISSN 13596454, doi: 10.1016/j.actamat.2003.10.026.

[15] E. C. Barnes, D. I. Wilson, M. L. Johns, Velocity profiling inside a ram extruder using magnetic resonance (MR) techniques, Chemical Engineering Science 61 (5) (2006) 1357-1367, ISSN 00092509, doi:10.1016/j.ces.2005.08.032.

[16] R. D. Wildman, S. Blackburn, D. M. Benton, P. A. McNeil, D. J. Parker, Investigation of paste flow using positron emission particle tracking, Powder Technology 103 (3) (1999) 220-229, ISSN 00325910, doi:10.1016/S0032-5910(99)00019-4.

[17] E. Mitsoulis, S. G. Hatzikiriakos, Steady flow simulations of compressible PTFE paste extrusion under severe wall slip, Journal of Non-Newtonian Fluid Mechanics 157 (1-2) (2009) 26-33, ISSN 03770257, doi:10.1016/j.jnnfm.2008.09.003.

[18] D. J. Horrobin, R. M. Nedderman, Die entry pressure drops in paste extrusion, Chemical Engineering Science 53 (18) (1998) 3215-3225, ISSN 00092509, doi:10.1016/S0009-2509(98)001055.

[19] J. J. Benbow, J. Bridgwater, Paste flow and extrusion, Oxford University Press, 1993.

[20] M. J. Patel, S. Blackburn, D. I. Wilson, Modelling of paste flows subject to liquid phase migration, International Journal for $\mathrm{Nu}$ merical Methods 72 (20) (2007) 1157-1180, doi:10.1002/nme.

[21] P. Jay, A. Magnin, J. M. Piau, Numerical simulation of viscoplastic fluid flows through an axisymmetric contraction, Journal of Fluids Engineering 124 (3) (2002) 700, ISSN 00982202, doi:10.1115/1.1486472.

[22] A. B. Ariawan, S. Ebnesajjad, S. G. Hatzikiriakos, Paste extrusion of polytetrafluoroethylene (PTFE) fine powder resins, The Canadian Journal of Chemical Engineering 80 (6) (2002) 1153-1165, ISSN 00084034, doi:10.1002/cjce.5450800617.

[23] A. Perrot, C. Lanos, Y. Melinge, P. Estellé, Mortar physical properties evolution in extrusion flow, Rheologica Acta 8 (2007) 1065-1073.

[24] M. Zhang, S. L. Rough, R. Ward, C. Seiler, D. I. Wilson, A comparison of ram extrusion by single-holed and multi-holed dies for extrusion-spheronisation of microcrystalline-based pastes., International Journal of Pharmaceutics 416 (1) (2011) 210-22, ISSN 1873-3476, doi:10.1016/j.ijpharm.2011.06.043.
[25] R. A. Basterfield, C. J. Lawrence, M. J. Adams, On the interpretation of orifice extrusion data for viscoplastic materials, Chemical Engineering Science 60 (10) (2005) 2599-2607, ISSN 00092509, doi:10.1016/j.ces.2004.12.019.

[26] C. A. Schneider, W. S. Rasband, K. W. Eliceiri, NIH Image to ImageJ: 25 years of image analysis, Nature Methods 9 (7) (2012) 671-675, ISSN 1548-7091, doi:10.1038/nmeth.2089.

[27] E. J. Dean, R. Glowinski, G. Guidoboni, On the numerical simulation of Bingham visco-plastic flow: Old and new results, Journal of Non Newtonian Fluid Mechanics 142 (1-3) (2007) 36-62, ISSN 0377-0257, doi: http://dx.doi.org/10.1016/j.jnnfm.2006.09.002.

[28] T. C. Papanastasiou, Flows of Materials with Yield, Journal of Rheology 31 (5) (1987) 385-404, ISSN 01486055, doi: $10.1122 / 1.549926$.

[29] E. Mitsoulis, Flows of viscoplastic materials: Models and computations, Rheology Reviews, British Society of Rheology 64 (2007) 135-178, ISSN 00457949.

[30] C. L. M. H. Navier, Memoire sur led lois du mouvement des fluides, Mem. Acad. Sci. Inst. France 6 (1823) 432-436.

[31] S. Mascia, M. J. Patel, S. Rough, P. J. Martin, D. Wilson, Liquid phase migration in the extrusion and squeezing of microcrystalline cellulose pastes., European journal of pharmaceutical sciences 29 (1) (2006) 22-34, ISSN 0928-0987, doi: 10.1016/j.ejps.2006.04.011.

[32] D.-C. Ko, B.-M. Kim, J.-C. Choi, Prediction of surface-fracture initiation in the axisymmetric extrusion and simple upsetting of an aluminum alloy, Journal of Materials Processing Technology 62 (1-3) (1996) 166-174, ISSN 09240136, doi:10.1016/09240136(95)02200-7.

[33] S. L. Rough, J. Bridgwater, D. I. Wilson, Effects of liquid phase migration on extrusion of microcrystalline cellulose pastes., International Journal of Pharmaceutics 204 (1-2) (2000) 117-26, ISSN 0378-5173.

[34] A. Perrot, C. Lanos, P. Estellé, Y. Melinge, Ram extrusion force for a frictional plastic material: model prediction and application to cement paste, Rheologica Acta 45 (4) (2006) 457-467, ISSN 0035-4511, doi:10.1007/s00397-005-0074-y.

[35] P. J. Martin, D. I. Wilson, P. E. Bonnett, Paste extrusion through non-axisymmetric geometries: Insights gained by application of a liquid phase drainage criterion, Powder Technology 168 (2) (2006) 64-73, ISSN 00325910, doi: 10.1016/j.powtec.2006.06.018.

[36] D. M. Kalyon, Apparent slip and viscoplasticity of concentrated suspensions, Journal of Rheology 49 (3) (2005) 621, ISSN 01486055, doi:10.1122/1.1879043.

[37] S. L. Rough, D. I. Wilson, J. Bridgwater, A model describing liquid phase migration within an extruding micro- 
crystalline cellulose paste, Chemical Engineering Research and Design 80 (7) (2002) 701-714, ISSN 02638762, doi: $10.1205 / 026387602320776786$.

[38] N. Roquet, P. Saramito, An adaptive finite element method for viscoplastic flows in a square pipe with stick slip at the wall, Journal of Non-Newtonian Fluid Mechanics 155 (3) (2008) 101115, ISSN 03770257, doi:10.1016/j.jnnfm.2007.12.003.

[39] E. E. Rosenbaum, S. G. Hatzikiriakos, Wall slip in the capillary flow of molten polymers subject to viscous heating, AIChE Journal 43 (3) (1997) 598-608, ISSN 0001-1541, doi: 10.1002/aic.690430305.

[40] Y. Damianou, M. Philippou, G. Kaoullas, G. C. Georgiou, Cessation of viscoplastic Poiseuille flow with wall slip, Journal of Non-Newtonian Fluid Mechanics 203 (2014) 24-37, ISSN 03770257, doi:10.1016/j.jnnfm.2013.10.004.

[41] M. A. Moyers-Gonzalez, I. A. Frigaard, Numerical solution of duct flows of multiple visco-plastic fluids, Journal of Non-Newtonian Fluid Mechanics 122 (2004) 227-241, doi: 10.1016/j.jnnfm.2003.12.010.

[42] A. J. Taylor, S. D. Wilson, Conduit flow of an incompressible, yield-stress fluid, Journal of Rheology 41 (1) (1997) 93, ISSN 01486055, doi:10.1122/1.550802.

[43] Y. Wang, Letter to the Editor: Comment on "Conduit flow of an incompressible, yield-stress fluid" [J. Rheol. 41, 93-101 (1997)], Journal of Rheology 41 (6) (1997) 1387, ISSN 01486055, doi: $10.1122 / 1.550847$.

[44] I. Frigaard, C. Nouar, On the usage of viscosity regularisation methods for visco-plastic fluid flow computation, Journal of Non-Newtonian Fluid Mechanics 127 (1) (2005) 1-26, ISSN 03770257, doi:10.1016/j.jnnfm.2005.01.003.

[45] P. Saramito, N. Roquet, An adaptive finite element method for viscoplastic fluid flows in pipes, Computer Methods in Applied Mechanics and Engineering 190 (2001) 5391-5412.

[46] R. R. Huilgol, Z. You, Application of the augmented Lagrangian method to steady pipe flows of Bingham, Casson and Herschel Bulkley fluids, Journal of Non-Newtonian Fluid Mechanics 128 (2-3) (2005) 126-143, ISSN 03770257, doi: $10.1016 / \mathrm{j} . j n n f m .2005 .04 .004$.

[47] L. Muravleva, E. Muravleva, G. C. Georgiou, E. Mitsoulis, Numerical simulations of cessation flows of a Bingham plastic with the augmented Lagrangian method, Journal of Non-Newtonian Fluid Mechanics 165 (9-10) (2010) 544-550, ISSN 03770257, doi:10.1016/j.jnnfm.2010.02.002. 
Revised manuscript LaTeX Source Files
Click here to download LaTeX Source Files: MBryanRevisedManuscriptLatexSource.zip

Revised manuscript LaTeX Source Files
Click here to download LaTeX Source Files: MBryanRevisedManuscriptLatexSource.zip 
Colour figures for inclusion on the web
Click here to download LaTeX Source Files: MBryanColourFiguresForWeb.zip

Colour figures for inclusion on the web
Click here to download LaTeX Source Files: MBryanColourFiguresForWeb.zip 
Information concerning figure widths

- Figure 1: 1-column

- Figure 2: 2-column (one sub-figure per column)

- Figure 3: 1-column

- Figure 4: 1.5-column (order is important, number of images per line is not)

- Figure 5: 1-column

- Figure 6: 1-column

- Figure 7: 1-column

- Figure 8: 1-column

- Figure 9: 1.5-column

- Figure 10: 2-column

- Figure 11: 1-column

- Figure A.1: 2-column

Colour versions of the photographs have been provided in the archive: MBryanColourFiguresForWeb.zip, for inclusion on the web only. The filenames are identical except for the removal of the word Gray from the end of each. 